\title{
CONTINUITY OF SOLUTIONS OF A NONLINEAR ELLIPTIC EQUATION
}

\author{
PierRe Bousquet ${ }^{1}$
}

\begin{abstract}
We consider a nonlinear elliptic equation of the form $\operatorname{div}[a(\nabla u)]+F[u]=0$ on a domain $\Omega$, subject to a Dirichlet boundary condition $\operatorname{tr} u=\phi$. We do not assume that the higher order term $a$ satisfies growth conditions from above. We prove the existence of continuous solutions either when $\Omega$ is convex and $\phi$ satisfies a one-sided bounded slope condition, or when $a$ is radial: $a(\xi)=\frac{l(|\xi|)}{|\xi|} \xi$ for some increasing $l: \mathbb{R}^{+} \rightarrow \mathbb{R}^{+}$.
\end{abstract}

Mathematics Subject Classification. 35J20, 35J25, 35J60.

Received March 11, 2011. Revised October 10, 2011.

Published online 16 January 2012.

\section{INTRODUCTION}

In this article, we consider the following nonlinear elliptic equation:

$$
\left\{\begin{array}{l}
\operatorname{div}[a(\nabla u)]+F[u]=0 \text { on } \Omega \\
u=\phi \text { on } \partial \Omega
\end{array}\right.
$$

Here, $\Omega$ is a bounded open Lipschitz set in $\mathbb{R}^{n}(n \geq 2)$ and $\phi: \partial \Omega \rightarrow \mathbb{R}$ is Lipschitz continuous. The vector field $a: \mathbb{R}^{n} \rightarrow \mathbb{R}^{n}$ is continuous and elliptic:

$$
\left\langle a(\xi)-a\left(\xi^{\prime}\right), \xi-\xi^{\prime}\right\rangle \geq 0 \quad \forall \xi, \xi^{\prime} \in \mathbb{R}^{n} .
$$

For $x \in \Omega$ and $u \in C^{0}(\bar{\Omega}), F[u](x)$ is a non linear functional of $u$. This term can be nonlocal. For instance, the following variational problem is considered in $[8]$ :

$$
\text { Minimize }\left\{\int_{\Omega} L(\nabla u(x)) \mathrm{d} x-\left(\int_{\Omega} h(x, u(x)) \mathrm{d} x\right)^{\beta}\right\} .
$$

The Euler equation can be written as in (1.1) with $a(\xi)=\nabla L(\xi)$ and

$$
F[u](x)=\beta\left(\int_{\Omega} h(x, u(x)) \mathrm{d} x\right)^{\beta-1} h_{u}(x, u(x)) .
$$

Keywords and phrases. Nonlinear elliptic equations, continuity of solutions, lower bounded slope condition, Lavrentiev phenomenon.

1 Université Aix-Marseille 1, LATP UMR6632 3 place Victor Hugo, 13331 Marseille Cedex 3, France. bousquet@cmi .univ-mrs.fr 
A solution of $(1.1)$ is a function $u \in W^{1,1}(\Omega)$ such that $a(\nabla u) \in L_{l o c}^{1}(\Omega), F[u] \in L_{l o c}^{1}(\Omega)$, tr $\left.u\right|_{\partial \Omega}=\phi$ and

$$
\text { (E) } \int_{\Omega}(\langle a(\nabla u(x)), \nabla \eta(x)\rangle-F[u](x) \eta(x)) \mathrm{d} x=0, \quad \forall \eta \in C_{c}^{\infty}(\Omega) .
$$

A natural approach to solve (1.1) is to consider it as a quasilinear elliptic equation to which Schauder's theory applies (see $[6,9]$ ). One then obtains a classical solution $u$. This requires however that $a$ belong to $C^{1, \alpha}(\Omega)$ and satisfies some structure conditions that we do not assume here.

Consider now the case when $a$ satisfies the following growth assumptions: there exists $p>1$ and $\alpha_{1}, \alpha_{2}$ and $\beta_{1}, \beta_{2}$ in $(0, \infty)$ such that

$$
\begin{aligned}
& \langle a(\xi), \xi\rangle \geq \alpha_{1}|\xi|^{p}-\beta_{1} \quad \forall \xi \in \mathbb{R}^{n}, \\
& |a(\xi)| \leq \alpha_{2}|\xi|^{p-1}+\beta_{2} \quad \forall \xi \in \mathbb{R}^{n} .
\end{aligned}
$$

Then, under suitable conditions on $F[u]$, there exists a solution to $(E)$ in $W^{1, p}(\Omega)$. This is the consequence of a theory initiated by Visik and then developped by many authors, notably Minty et al. (see [10] and the references therein). Once the existence of a $W^{1, p}$ solution $u$ is established, the question of the regularity of $u$ arises. Is the solution $C^{1}(\Omega)$ or even $C^{2}(\Omega)$, so that equation (1.1) is satisfied in a classical sense? Is the solution continuous up to the boundary of $\Omega$, so that the trace is a 'true' restriction to $\partial \Omega$ ?

Another way to prove the existence of classical solutions of $(E)$ has been considered by Hartman and Stampacchia. In [8], they proved the existence of regular solutions to $(E)$ without assuming any growth assumption from above on $a$. Here, 'regular' means Lipschitz continuous. This is the key regularity property from which we may deduce further regularity when the coefficients of the equation are smooth (see [8], Sect. 14).

We proceed to detail the strategy of Hartman and Stampacchia. We first introduce for $K>0$ the set $\operatorname{Lip}_{\phi}(\Omega, K)$ of those functions $u: \Omega \rightarrow \mathbb{R}$ which are Lipschitz continuous on $\Omega$, their Lipschitz rank being not larger than $K$. This set is not empty except when $K$ is lower than the Lipschitz rank $K_{\phi}$ of $\phi$. We also denote by $\operatorname{Lip}_{\phi}(\Omega)$ the set of Lipschitz continuous functions on $\Omega$. The set $C^{0}(\bar{\Omega})$ is endowed with the $L^{\infty}$ norm. For $K \geq K_{\phi}$, we say that $u_{K} \in \operatorname{Lip}_{\phi}(\Omega, K)$ is a $K$ quasi solution of $(E)$ if

$$
\int_{\Omega}\left\langle a\left(\nabla u_{K}\right), \nabla\left(v-u_{K}\right)\right\rangle-F\left[u_{K}\right]\left(v-u_{K}\right) \geq 0, \quad \forall v \in \operatorname{Lip}_{\phi}(\Omega, K) .
$$

When $a$ satisfies (1.2), there exists a $K$ quasi solution $u_{K}$ for each $K \geq K_{\phi}$ ([8] Thm. 1.1). Under a stronger ellipticity condition on $a$, Hartman and Stampacchia prove that there exists $C>0$ such that for any $K \geq K_{\phi}$,

$$
\left\|u_{K}\right\|_{L^{\infty}(\Omega)}+\left\|\nabla u_{K}\right\|_{L^{\infty}(\Omega)} \leq C .
$$

In order to obtain such an estimate without any growth assumption from above on $a, \phi$ is required to satisfy the bounded slope condition: there exists $Q>0$ such that for any $\gamma \in \partial \Omega$, there exist $\zeta_{\gamma}^{-}$and $\zeta_{\gamma}^{+}$in $\mathbb{R}^{n}$ which satisfy $\left|\zeta_{\gamma}^{ \pm}\right| \leq Q$ and

$$
\phi(\gamma)+\left\langle\zeta_{\gamma}^{-}, y-\gamma\right\rangle \leq \phi(y) \leq \phi(\gamma)+\left\langle\zeta_{\gamma}^{+}, y-\gamma\right\rangle, \quad \forall y \in \partial \Omega .
$$

By using (1.6), one can extract from $\left(u_{K}\right)_{K \geq K_{\phi}}$ a subsequence which converges to a Lipschitz solution of $(E)$.

In [2], we have generalized this result to a larger class of functions $\phi$. The bounded slope condition used in [8] is indeed quite restrictive. It requires that $\Omega$ be convex (except when $\phi$ is affine). It forces $\phi$ to be affine on 'flat parts' of $\partial \Omega$. Moreover, if $\Omega$ is smooth, then $\phi$ must be smooth as well (see Hartman [7] for precise statements; see also [1]). Recently, Clarke [4] has introduced a new hypothesis on $\phi$, the lower bounded slope condition of rank $Q(Q>0)$ : given any point $\gamma \in \partial \Omega$, there exists an affine function

$$
y \mapsto\left\langle\zeta_{\gamma}, y-\gamma\right\rangle+\phi(\gamma)
$$


with $\left|\zeta_{\gamma}\right| \leq Q$ such that

$$
\left\langle\zeta_{\gamma}, y-\gamma\right\rangle+\phi(\gamma) \leq \phi(y), \quad \forall y \in \partial \Omega
$$

The map $\phi: \partial \Omega \rightarrow \mathbb{R}$ satisfies the lower bounded slope condition if and only if $\phi$ is the restriction to $\partial \Omega$ of a convex function defined on $\mathbb{R}^{n}$. When $\Omega$ is uniformly convex, $\phi$ satisfies the lower bounded slope condition if and only if it is the restriction to $\partial \Omega$ of a semiconvex function (see [1] for details and further properties).

When $\phi$ satisfies the lower bounded slope condition (rather than the full two-sided bounded slope condition), it can be proved (see [2]) that a solution $u$ of $(E)$ still exists in $W^{1,2}(\Omega) \cap W_{l o c}^{1, \infty}(\Omega)$ when $a$ satisfies

$$
\left\langle a(\xi)-a\left(\xi^{\prime}\right), \xi-\xi^{\prime}\right\rangle \geq \mu\left|\xi-\xi^{\prime}\right|^{2}
$$

and $F$ satisfies growth assumptions similar to those of [8]. The convexity of $\Omega$ is also required here. The main idea of the proof, inspired from [4], was that a 'one sided barrier' is enough to obtain local Lipschitz continuity. Moreover, the result was optimal in the following sense: even when $a(p)=p, F[u]=0$ and $\Omega$ is a disk in $\mathbb{R}^{2}$, it may happen that the corresponding solution is not globally Lipschitz on $\Omega$ if $\phi$ satisfies the mere lower bounded slope condition.

In [2], the solution that we obtained satisfied the boundary condition only in the sense of traces. We were unable at that time to prove the continuity of the solution up to the boundary, except when $\Omega$ was a polyhedron. This is the content of our first main result Theorem 2.3 below to generalize this property to any convex domains (under the same assumptions). As in [3], the proof uses 'implicit barriers'. In contrast with classical barriers which are explicitly defined in terms of the distance to the boundary and the function $\phi$ (see e.g. $[6,8]$ ), the implicit barriers are obtained as solutions of auxiliary problems stated on larger domains $\Omega_{0} \supset \Omega$ with different boundary conditions.

Up to now, only convex domains have been considered. It is an open problem to know whether Theorem 2.3 holds true on any smooth domain, even when $\phi$ is smooth. However, we prove in Theorem 2.5 that the Lipschitz continuity of $\phi$ is enough to prove the existence of Hölder continuous solutions when $a$ is radial: there exists $l: \mathbb{R}^{+} \rightarrow \mathbb{R}^{+}$such that $a(\xi)=\frac{l(|\xi|)}{|\xi|} \xi$, where $|\cdot|$ is the Euclidean norm in $\mathbb{R}^{n}$. Once again, we only assume that $l$ satisfies a growth assumption from below which corresponds to (1.8).

The next section describes the hypotheses that we posit on the data. Each of the following sections is devoted to the proof of Theorems 2.3 and 2.5 respectively.

\section{MAIN RESULtS}

Throughout the paper, $\Omega$ is a bounded open Lipschitz set. We denote by $\Gamma$ the boundary of $\Omega$. Hence, there is a $\delta>0$ such that for every point $\gamma \in \Gamma, \Gamma \cap B(\gamma, \delta)$ is the graph of a Lipschitz function (in an appropriate coordinate system varying with $\gamma$ ). We also assume that the map $\phi: \Gamma \rightarrow \mathbb{R}$ is Lipschitz continuous of rank $K_{\phi}$.

For the sake of clarity, we proceed to quote some results from [8]. Assume that $F: C^{0}(\bar{\Omega}) \rightarrow L^{1}(\Omega)$ is continuous:

$$
\begin{aligned}
& \text { If } u_{h} \in C^{0}(\bar{\Omega}) \text { for } h=1,2, \ldots \text { converges uniformly to } u \\
& \text { on } \bar{\Omega} \text { as } h \rightarrow \infty \text {, then } F\left[u_{h}\right] \rightarrow F[u] \text { in } L^{1}(\Omega) \text {. }
\end{aligned}
$$

We also assume that $F$ is locally bounded: for every $M>0$, there exists $\chi(M)>0$ such that

$$
|u(x)| \leq M \text { on } \Omega \Rightarrow|F[u](x)| \leq \chi(M) .
$$

The existence of quasi solutions follows from

Theorem 2.1 ([8] Lem. 12.1). Assume that a is continuous and elliptic (see (1.2)), and that $F$ satisfies (HF1) and (HF2). Then for each $K \geq K_{\phi}$, there exists at least one $u_{K} \in \operatorname{Lip}_{\phi}(\Omega, K)$ such that

$$
\int_{\Omega}\left\langle a\left(\nabla u_{K}\right), \nabla\left(v-u_{K}\right)\right\rangle-F\left[u_{K}\right]\left(v-u_{K}\right) \geq 0, \quad \forall v \in \operatorname{Lip}_{\phi}(\Omega, K) .
$$


The a priori $L^{\infty}$ bound on quasi solutions can be obtained under the following assumptions (see [8] for more general conditions): we assume that $a \in C^{0}\left(\mathbb{R}^{n}, \mathbb{R}^{n}\right)$ satisfy (1.3) for some $1<p \leq n$, and that

$$
(H F 3) \quad F[u](x) \operatorname{sgn} u(x) \leq c \sum_{i=1}^{m}\|u\|_{L^{\alpha(i)}(\Omega)}^{\beta(i)}|u(x)|^{\gamma(i)-1}, \quad x \in \Omega \text { a.e. }
$$

where $c \geq 0, \alpha(i) \geq 1, \beta(i) \geq 0, \gamma(i) \geq 1$ and $\alpha(i) \leq p^{*}, \beta(i)+\gamma(i)<p$. Here, $p^{*}=n p /(n-p)$ if $n>p$. When $n \leq p$, we replace the condition $\alpha(i) \leq p^{*}$ by $\alpha(i)<\infty$.

We then have

Theorem 2.2 ([8], Thm. 8.1). There exists a constant $T$ such that for any $K \geq K_{\phi}$, for any $K$ quasi solution $u$, we have

$$
\|u\|_{L^{\infty}(\Omega)} \leq T .
$$

The constant $T$ depends on $|\Omega|,\|\phi\|_{L^{\infty}(\Omega)}$ and the parameters in (1.3) and (HF3).

For later use, we observe that (1.8) implies (1.3) for any $p \leq 2$ (for $p=2$, one can take e.g. $\alpha_{1}=\mu / 2$ and $\left.\beta_{1}=|a(0)|^{2} /(2 \mu)\right)$.

We can now state our first main result:

Theorem 2.3. Assume that $\Omega$ is convex, $\phi$ satisfies the lower bounded slope condition as in (1.7), and $a \in$ $C^{0}\left(\mathbb{R}^{n}, \mathbb{R}^{n}\right)$ is uniformly elliptic as in (1.8). If $F$ satisfies (HF1), (HF2) and (HF3) with $p=2$, then there exists a solution u to $(E)$ in $W^{1,2}(\Omega) \cap L^{\infty}(\Omega)$ which is locally Lipschitz in $\Omega$. Moreover, $u$ is Hölder continuous on $\bar{\Omega}$ and agrees with $\phi$ on $\partial \Omega$.

As explained in the introduction, the first sentence of the above statement is proved in [2]. The continuity of $u$ is established in Section 3 below.

In the class of those functions which are locally Lipschitz on $\Omega$ and continuous up to the boundary, a uniqueness result can be stated provided that a further condition is introduced on $F[u]$ regarding its monotonicity.

Theorem 2.4. In addition to the assumptions of Theorem 2.3, assume that for any $u_{1}, u_{2} \in C^{0}(\bar{\Omega})$, we have

$$
\int_{\Omega}\left(F\left[u_{1}\right]-F\left[u_{2}\right]\right)\left(u_{1}-u_{2}\right) \leq 0
$$

Then there exists one and only one locally Lipschitz solution $u$ to the equation $(E)$ which is continuous on the closure of $\Omega$.

Theorem 2.4 is proved at the end of Section 3.

When the domain $\Omega$ is not necessarily convex or when the Lipschitz function $\phi$ does not satisfy a one sided bounded slope condition, it is still possible to prove the existence of a continuous solution when $a$ is radial:

Theorem 2.5. Assume that $a \in C^{0}\left(\mathbb{R}^{n}, \mathbb{R}^{n}\right)$ can be written as $a(\xi)=\frac{l(|\xi|)}{|\xi|} \xi$ where $l: \mathbb{R}^{+} \rightarrow \mathbb{R}^{+}$satisfies:

(Hl) there exists $\mu>0$ and $p \geq 2$ such that for any $0<s<t$,

$$
l(t)-l(s) \geq \mu(t-s)^{p-1} .
$$

Assume that $F$ satisfies ( $H F 1),(H F 2)$ and ( $H F 3)$ (for the same exponent $p$ ). If $\Omega$ has the uniform exterior sphere condition and $\phi$ is Lipschitz continuous, then there exists a solution $u$ of $(E)$ in $W^{1, p}(\Omega)$ which is Hölder continuous on $\bar{\Omega}$. 
Please remember that a solution $u$ is such that $a(\nabla u)$ and $F[u]$ belong to $L_{l o c}^{1}(\Omega),\left.\operatorname{tr} u\right|_{\partial \Omega}=\phi$ and $(E)$ is satisfied. We say that $\Omega$ has the uniform exterior sphere condition if there exists $r>0$ such that for any $\gamma \in \Gamma$, there exists $z \in \mathbb{R}^{n}$ which satisfies:

(1) $|z-\gamma|=r$;

(2) $B(z, r) \subset \mathbb{R}^{n} \backslash \Omega$.

Since $a$ is continuous, we necessarily have $l(0)=0$.

One of the most classical examples of radial fields a satisfying the above assumptions is the $p$ Laplacian $(p \geq 2): a(\xi)=|\xi|^{p-2} \xi$. More generally, any $C^{1}$ map $l$ such that $\inf _{t>0} \frac{l^{\prime}(t)}{t^{p-2}}>0$ satisfies $(H l)$.

\section{Proof of Theorem 2.3}

Since $\phi$ satisfies the lower bounded slope condition, it is the restriction to $\Gamma$ of a convex function which is globally Lipschitz on $\mathbb{R}^{n}$ (see [1]). We still denote by $\phi$ this extension and by $K_{\phi}$ its Lipschitz rank on $\mathbb{R}^{n}$.

By Theorem 2.1, for every $K \geq K_{\phi}$, there exists a $K$ quasi solution to (1.5). By Theorem 2.2 and $(H F 2)$, there exists a constant $T$ (independent of $K$ ) such that if $u$ is a $K$ quasi solution, then

$$
\|u\|_{L^{\infty}(\Omega)} \leq T, \quad\|F[u]\|_{L^{\infty}(\Omega)} \leq \chi(T) .
$$

It then easily follows from (1.8) that there exists $S>0$ such that $\|u\|_{W^{1,2}(\Omega)} \leq S$ for any $K$ quasi solution (see [2], Prop. 3.3 for details). Here, $S$ depends on $\|\phi\|_{W^{1, \infty}(\Omega)},\|a(\nabla \phi)\|_{L^{\infty}(\Omega)},|\Omega|, \mu, T$ and $\chi(T)$.

Barriers are the basic tool to control the behaviour of quasi solutions near the boundary.

Definition 3.1. Let $K_{0} \geq K_{\phi}$ and $\gamma \in \partial \Omega$. Then we say that a function $w: \Omega \rightarrow \mathbb{R}$ is a lower barrier for $(E)$ at $\gamma$ in $\operatorname{Lip}_{\phi}\left(\Omega, K_{0}\right)$ if the following properties are satisfied:

- the function $w$ is Lipschitz continuous on $\Omega$ of rank $\leq K_{0}$;

- $w(\gamma)=\phi(\gamma)$ and $w \leq \phi$ on $\partial \Omega$;

- for $K \geq K_{0}$, any $K$ quasi solution $u$ of $(E)$ satisfies $u \geq w$ on $\Omega$.

We define similarly an upper barrier. We can construct lower barriers for $K$ quasi solutions by using the lower bounded slope condition (see [2], Prop. 3.4):

Proposition 3.2. There exists $K_{0} \geq K_{\phi}$ such that for any $\gamma \in \Gamma$, there exists a lower barrier $w$ in $\operatorname{Lip}_{\phi}\left(\Omega, K_{0}\right)$.

This lower barrier was used to prove the following key estimate (see [2], inequality (3.10)):

Proposition 3.3. There exists $Q \geq 0$ such that for any $K \geq K_{\phi}$, for any $K$ quasi solution $u$, we have

$$
u(x) \leq u(y)+Q \frac{|x-y|}{\left|x-\pi_{\Gamma}(x \mid y)\right|}, \quad x, y \in \Omega .
$$

Here $\pi_{\Gamma}(x \mid y)$ denotes the unique point of $\Gamma$ of the form $x+t(y-x), t>0$.

The constants $K_{0}$ in Proposition 3.2 and $Q$ in Proposition 3.3 depend on $\mu, T, \chi(T),\|\phi\|_{L^{\infty}(\Omega)}, K_{\phi}$ and the diameter diam $\Omega$ of $\Omega$. Moreover, (3.1) implies that on any compact subset $\Omega_{0} \subset \Omega$, the Lipschitz rank of $u_{K}$ is bounded by $Q / \mathrm{d}\left(\Omega_{0}, \Gamma\right)$ (here, $\mathrm{d}\left(\Omega_{0}, \Gamma\right)$ denotes the distance between $\Omega_{0}$ and $\Gamma$ ).

The new result of this section is given by the following proposition:

Proposition 3.4. There exists $C>0$ such that for any $K \geq K_{\phi}$, for any $K$ quasi solution $u$, we have

$$
|u(x)-u(y)| \leq C|x-y|^{\alpha} \quad x, y \in \Omega,
$$

where $\alpha:=\frac{1}{n+1}$. 
Proof of Proposition 3.4. Estimate (3.2) will follow from

Lemma 3.5. [3] Let $u \in W^{1, p}\left((-1,1)^{n}\right), p>1$. We assume that there exists $Q>0$ such that for a.e. $t \in(-1,1)$, for a.e. $x_{1}^{\prime}, x_{2}^{\prime} \in(-1,1)^{n-1}$,

$$
\left|u\left(x_{1}^{\prime}, t\right)-u\left(x_{2}^{\prime}, t\right)\right| \leq Q\left|x_{1}^{\prime}-x_{2}^{\prime}\right| .
$$

Then there exists $C>0$ only depending on $n, p, Q$ and $\left\|\partial_{t} u\right\|_{L^{p}\left((-1,1)^{n}\right)}$ such that for some representative $\tilde{u}$ of $u$, we have for any $x_{1}, x_{2} \in(-1,1)^{n}$,

$$
\left|\tilde{u}\left(x_{1}\right)-\tilde{u}\left(x_{2}\right)\right| \leq C\left|x_{1}-x_{2}\right|^{\frac{p-1}{n-1+p}} .
$$

Proof. By reflection and regularisation, we can assume that $u$ is the restriction to $(-1,1)^{n}$ of a map in $C^{\infty}\left(\mathbb{R}^{n}\right)$ still denoted by $u$. We introduce a smooth kernel $\rho \in C_{c}^{\infty}\left(\mathbb{R}^{n-1}, \mathbb{R}^{+}\right), \int_{\mathbb{R}^{n-1}} \rho=1$, supp $\rho \subset B^{n-1}(0,1)$. We denote by $\rho_{\epsilon}$ the function defined by $\rho_{\epsilon}(\cdot)=\rho(\cdot / \epsilon) / \epsilon^{n-1}$. We consider

$$
u_{\epsilon}\left(x^{\prime}, t\right)=\int_{\mathbb{R}^{n-1}} u\left(x^{\prime}-y^{\prime}, t\right) \rho_{\epsilon}\left(y^{\prime}\right) \mathrm{d} y^{\prime} .
$$

By (3.3), we have $\left|u_{\epsilon}\left(x^{\prime}, t\right)-u\left(x^{\prime}, t\right)\right| \leq Q \epsilon$.

Let $x^{\prime} \in(-1,1)^{n-1}$ and $-1<t_{1}<t_{2}<1$. Then

$$
\begin{aligned}
\left|u\left(x^{\prime}, t_{1}\right)-u\left(x^{\prime}, t_{2}\right)\right| & \leq\left|u\left(x^{\prime}, t_{1}\right)-u_{\epsilon}\left(x^{\prime}, t_{1}\right)\right|+\left|u_{\epsilon}\left(x^{\prime}, t_{1}\right)-u_{\epsilon}\left(x^{\prime}, t_{2}\right)\right|+\left|u_{\epsilon}\left(x^{\prime}, t_{2}\right)-u\left(x^{\prime}, t_{2}\right)\right| \\
& \leq 2 \epsilon Q+\int_{\mathbb{R}^{n-1}} \rho_{\epsilon}\left(x^{\prime}-y^{\prime}\right) \mathrm{d} y^{\prime} \int_{t_{1}}^{t_{2}}\left|\partial_{t} u\left(y^{\prime}, t\right)\right| \mathrm{d} t .
\end{aligned}
$$

By Hölder's inequality, we get

$$
\int_{t_{1}}^{t_{2}} \mathrm{~d} t \int_{\mathbb{R}^{n-1}} \rho_{\epsilon}\left(x^{\prime}-y^{\prime}\right)\left|\partial_{t} u\left(y^{\prime}, t\right)\right| \mathrm{d} y^{\prime} \leq\left\|\partial_{t} u\right\|_{L^{p}\left(B^{n-1}\left(x^{\prime}, \epsilon\right) \times(-1,1)\right)}\left|t_{2}-t_{1}\right|^{1-\frac{1}{p}} \frac{\|\rho\|_{L^{p^{\prime}\left(\mathbb{R}^{n-1}\right)}}}{\epsilon^{\frac{n-1}{p}}} .
$$

Therefore,

$$
\left|u\left(x^{\prime}, t_{1}\right)-u\left(x^{\prime}, t_{2}\right)\right| \leq 2 \epsilon Q+\frac{\|\rho\|_{L^{p^{\prime}}\left(\mathbb{R}^{n-1}\right)}}{\epsilon^{\frac{n-1}{p}}}\left\|\partial_{t} u\right\|_{L^{p}\left(B^{n-1}\left(x^{\prime}, \epsilon\right) \times(-1,1)\right)}\left|t_{2}-t_{1}\right|^{1-\frac{1}{p}} .
$$

We now take $\epsilon:=\left|t_{2}-t_{1}\right|^{\frac{p-1}{n-1+p}}$. This gives

$$
\left|u\left(x^{\prime}, t_{1}\right)-u\left(x^{\prime}, t_{2}\right)\right| \leq C\left|t_{2}-t_{1}\right|^{\frac{p-1}{n-1+p}}
$$

where $C$ depends only on $n, p, Q$ and $\left\|\partial_{t} u\right\|_{L^{p}\left((-1,1)^{n}\right)}$. Lemma 3.5 follows from (3.3) and (3.4).

We observe that the possibility to exploit the continuity of a map in one direction together with the integrability properties in the other directions had already been used in [4].

The construction of upper barriers will be based on:

Lemma 3.6. Let $\Omega^{*}$ be a bounded subset of $\mathbb{R}^{n}$ such that $\Omega^{*} \supset \Omega$. Let $\phi^{*}: \mathbb{R}^{n} \rightarrow \mathbb{R}$ be a convex function such that $\left.\phi^{*}\right|_{\Omega^{*}} \in C^{\infty}\left(\Omega^{*}\right)$. We assume that $\phi^{*} \geq \phi$ on $\Gamma$. Let $u^{*} \in \operatorname{Lip}_{\phi^{*}}\left(\Omega^{*}, K\right)$ be a $K$ quasi solution in $\operatorname{Lip}_{\phi^{*}}\left(\Omega^{*}, K\right)$ :

$$
\int_{\Omega^{*}}\left\langle a\left(\nabla u^{*}\right), \nabla\left(v-u^{*}\right)\right\rangle-\chi(T)\left(v-u^{*}\right) \geq 0 \quad, \quad \forall v \in \operatorname{Lip}_{\phi^{*}}\left(\Omega^{*}, K\right) .
$$

Then for any $K$ quasi solution $u$ of $(E)$ in $\operatorname{Lip}_{\phi}(\Omega, K), u^{*} \geq u$ on $\Omega$. 
(The constant $\chi(T)>0$ has been introduced at the beginning of Section $3:|F[u]| \leq \chi(T)$ for any $K$ quasi solution $u$ of $(E)$ in $\left.\operatorname{Lip}_{\phi}(\Omega, K)\right)$.

Proof. We first prove that $\phi^{*} \leq u^{*}$ on $\Omega^{*}$ by inserting $v(x):=\max \left(u^{*}(x), \phi^{*}(x)\right)$ in (3.5). This gives

$$
\int_{\left[\phi^{*}>u^{*}\right]}\left\langle a\left(\nabla u^{*}\right), \nabla \phi^{*}-\nabla u^{*}\right\rangle-\chi(T)\left(\phi^{*}-u^{*}\right) \geq 0 .
$$

Using (1.8), we obtain

$$
\int_{\left[\phi^{*}>u^{*}\right]}\left\langle a\left(\nabla \phi^{*}\right), \nabla u^{*}-\nabla \phi^{*}\right\rangle \leq-\chi(T) \int_{\left[\phi^{*}>u^{*}\right]}\left(\phi^{*}-u^{*}\right) .
$$

We claim that the left hand side is non negative.

Indeed, let $\left\{a_{\epsilon}=\left(a_{\epsilon}^{1}, \ldots, a_{\epsilon}^{n}\right)\right\}_{\epsilon>0}$ be a family of smooth vector fields which converge locally uniformly to $a$ and satisfy (1.8) with the same $\mu$ (a convolution of $a$ by a smooth kernel will do). For each $\epsilon$, Stokes formula implies

$$
\begin{aligned}
\int_{\left[\phi^{*}>u^{*}\right]}\left\langle a_{\epsilon}\left(\nabla \phi^{*}\right), \nabla u^{*}-\nabla \phi^{*}\right\rangle & =\int_{\left[\phi^{*}>u^{*}\right]} \operatorname{div}\left[a_{\epsilon}\left(\nabla \phi^{*}\right)\right]\left(\phi^{*}-u^{*}\right) \\
& =\int_{\left[\phi^{*}>u^{*}\right]} \sum_{i, j=1}^{n} \frac{\partial a_{\epsilon}^{i}}{\partial \xi_{j}}\left(\nabla \phi^{*}\right) \frac{\partial^{2} \phi^{*}}{\partial x_{i} \partial x_{j}}\left(\phi^{*}-u^{*}\right) \geq 0,
\end{aligned}
$$

by convexity of $\phi^{*}$. By letting $\epsilon \rightarrow 0$, we get

$$
\int_{\left[\phi^{*}>u^{*}\right]}\left\langle a\left(\nabla \phi^{*}\right), \nabla u^{*}-\nabla \phi^{*}\right\rangle \geq 0
$$

The comparison of (3.6) and (3.7) implies that $\phi^{*} \leq u^{*}$ on $\overline{\Omega^{*}}$.

In particular, $u^{*} \geq \phi^{*} \geq \phi$ on $\Gamma$. We now prove that $u^{*} \geq u$ on $\bar{\Omega}$, for any $K$ quasi solution $u$ of $(E)$ in $\operatorname{Lip}_{\phi}(\Omega, K)$. The function

$$
v(x):=\min \left(u, u^{*}\right)(x)
$$

belongs to $\operatorname{Lip}_{\phi}(\Omega, K)$. The function

$$
v^{*}(x):=\left\{\begin{array}{l}
\max \left(u, u^{*}\right)(x) \text { if } x \in \Omega, \\
u^{*}(x) \text { otherwise }
\end{array}\right.
$$

belongs to $\operatorname{Lip}\left(\Omega^{*}, K\right)$ and agrees with $u^{*}$ on $\partial \Omega^{*}$.

By inserting $v$ in (1.5) and $v^{*}$ in (3.5), we get

$$
\begin{gathered}
\int_{\left[u>u^{*}\right]}\left(\left\langle a(\nabla u(x)), \nabla u^{*}(x)-\nabla u(x)\right\rangle-F[u]\left(u^{*}(x)-u(x)\right)\right) \mathrm{d} x \geq 0, \\
\int_{\left[u>u^{*}\right]}\left(\left\langle a\left(\nabla u^{*}(x)\right), \nabla u(x)-\nabla u^{*}(x)\right\rangle-\chi(T)\left(u(x)-u^{*}(x)\right)\right) \mathrm{d} x \geq 0 .
\end{gathered}
$$

This gives by (1.8) and the definition of $\chi(T)$

$$
\begin{aligned}
\mu \int_{\left[u>u^{*}\right]}\left|\nabla u-\nabla u^{*}\right|^{2} & \leq \int_{\left[u>u^{*}\right]}\left\langle a(\nabla u)-a\left(\nabla u^{*}\right), \nabla u-\nabla u^{*}\right\rangle \\
& \leq \int_{\left[u>u^{*}\right]}(F[u]-\chi(T))\left(u-u^{*}\right) \leq 0,
\end{aligned}
$$

which implies that $u \leq u^{*}$ on $\Omega$. This completes the proof of Lemma 3.6. 
A first consequence of Lemma 3.6 is given by:

Lemma 3.7. There exists $C_{1}>0$ such that for any $K \geq K_{\phi}$, any $K$ quasi solution $u$ and any $\gamma \in \Gamma, x \in \Omega$, we have

$$
u(x) \leq \phi(\gamma)+C_{1}|x-\gamma|^{\alpha},
$$

where $\alpha=\frac{1}{n+1}$.

Proof. Fix $\gamma \in \Gamma$. Since $\Omega$ is convex, there exists an open hypercube $\Omega^{*}$ such that $\Omega^{*} \supset \Omega$ and $\gamma$ is the center of an $n-1$ dimensional face $\Sigma$ of $\Omega^{*}$. We introduce

$$
\phi^{*}(x):=\phi(\gamma)+K_{\phi}|x-\gamma| .
$$

Thus, $\phi^{*} \geq \phi$ on $\Gamma$.

By Theorem 2.1, for any $K \geq K_{\phi}$, there exists a $K$ quasi solution $u^{*} \in \operatorname{Lip}_{\phi^{*}}\left(\Omega^{*}, K\right)$ satisfying (3.5). Moreover, there exist $T^{*}>0, S^{*}>0$ (not depending on $K$ ) such that

$$
\left\|u^{*}\right\|_{L^{\infty}\left(\Omega^{*}\right)} \leq T^{*}, \quad\left\|u^{*}\right\|_{W^{1,2}\left(\Omega^{*}\right)} \leq S^{*} .
$$

By Proposition 3.3, there exists $Q^{*}>0$ (not depending on $K$ ) such that

$$
u^{*}(x) \leq u^{*}(y)+Q^{*} \frac{|x-y|}{\left|x-\pi_{\partial \Omega^{*}}(x \mid y)\right|}, \quad x, y \in \Omega^{*} .
$$

Let $\Omega_{1}^{*}=\frac{1}{2}\left(\Omega^{*}-\gamma\right)+\gamma$. In view of $(3.9)$, we can apply Lemma 3.5 on $\Omega_{1}^{*}$ with $p=2$. We get

$$
\left|u^{*}(x)-u^{*}(\gamma)\right| \leq C_{1}|x-\gamma|^{\alpha}, \quad x \in \Omega_{1}^{*}
$$

for some $C_{1}>0$. By enlarging $C_{1}$ if necessary, we can assume that this inequality holds true for any $x \in \Omega^{*}$.

By Lemma 3.6 and the fact that $u^{*}(\gamma)=\phi^{*}(\gamma)=\phi(\gamma)$, we have

$$
u(x) \leq u^{*}(x) \leq \phi(\gamma)+C_{1}|x-\gamma|^{\alpha}, \quad x \in \Omega .
$$

This completes the proof of Lemma 3.7.

In order to exploit the estimate given by Lemma 3.7, we need the following maximum principle:

Lemma 3.8. There exists $C_{0}>0$ such that for any $K \geq K_{\phi}$, any $K$ quasi solution $u$ and any $x, y \in \Omega$,

$$
|u(x)-u(y)| \leq \max _{\substack{z \in \Omega, \gamma \in \Gamma,|z-\gamma| \leq|x-y|}}|u(z)-\phi(\gamma)|+C_{0}|x-y| .
$$

The constant $C_{0}$ depends only on $\chi(T) / \mu$, and $|\Omega|$. Lemma 3.8 is a consequence of the proof of [8] Lemma 10.0 (more precisely, it is a rephrasing of inequality (10.14) there). The lower bounded slope condition plays no role here, neither does the convexity of $\Omega$.

We now complete the proof of Proposition 3.4. For any $K \geq K_{\phi}$ and any $K$ quasi solution $u$, we have by Proposition 3.2

$$
u(z) \geq w(x) \geq \phi(\gamma)-K_{0}|z-\gamma|, \quad \gamma \in \Gamma, z \in \Omega .
$$

This gives a lower bound of $u(z)-\phi(\gamma)$ when $z \in \Omega$.

From (3.12) and (3.8), for $K \geq K_{\phi}$, a $K$ quasi solution $u$ satisfies

$$
\max _{\substack{z \in \Omega, \gamma \in \Gamma,|z-\gamma| \leq|x-y|}}|u(z)-\phi(\gamma)| \leq\left(K_{0}(\operatorname{diam} \Omega)^{1-\alpha}+C_{1}\right)|x-y|^{\alpha} .
$$

By Lemma 3.8, this implies that for any $x, y \in \Omega$,

$$
|u(x)-u(y)| \leq C|x-y|^{\alpha},
$$

with $C:=\left(K_{0}+C_{0}\right)(\operatorname{diam} \Omega)^{1-\alpha}+C_{1}$. Hence, $u$ is Hölder continuous. Proposition 3.4 follows at once. 
Finally, we complete the proof of Theorem 2.3. For each $K \geq K_{\phi}$, let $u_{K} \in \operatorname{Lip}_{\phi}(\Omega, K)$ a $K$ quasi solution, so that $\left\|u_{K}\right\|_{L^{\infty}(\Omega)} \leq T,\left\|u_{K}\right\|_{W^{1,2}(\Omega)} \leq S$ and the Lipschitz rank of $u_{K}$ on any compact subset $\Omega_{0} \subset \Omega$ is bounded by $Q / \mathrm{d}\left(\Omega_{0}, \Gamma\right)$. Finally, $(3.2)$ holds true for any $u_{K}$.

Then there exists a subsequence $\left(u_{K_{i}}\right)$ which uniformly converges on $\bar{\Omega}$ to a function $u$ which is locally Lipschitz on $\Omega$, and Hölder continuous on $\bar{\Omega}$ of order $\alpha$. As in [2], Proposition 3.6, one can also prove that the function $u$ is a solution of $(E)$. This completes the proof of Theorem 2.3.

Proof of Theorem 2.4. Here, we assume further that if $u_{1}, u_{2}$ are two continuous functions on $\bar{\Omega}$, then

$$
\int_{\Omega}\left(F\left[u_{1}\right](x)-F\left[u_{2}\right](x)\right)\left(u_{1}(x)-u_{2}(x)\right) \mathrm{d} x \leq 0 .
$$

Let $u_{1}, u_{2}: \Omega \rightarrow \mathbb{R}$ be such that:

(1) $u_{1}, u_{2}$ are locally Lipschitz on $\Omega$;

(2) $u_{1}, u_{2}$ are continuous on $\bar{\Omega}$ and agree with $\phi$ on $\Gamma$;

(3) $u_{1}, u_{2}$ are solutions of $(E)$.

We then prove that $u_{1}=u_{2}$. Let $\theta: \mathbb{R} \rightarrow \mathbb{R}$ be a smooth odd nondecreasing function such that

$$
\theta(t)= \begin{cases}0 & \text { if }|t| \leq 1 \\ t & \text { if }|t| \geq 2 .\end{cases}
$$

We define for each $i \geq 1, \theta_{i}(t)=\theta(i t) / i$ and $\eta_{i}(x)=\theta_{i}\left(u_{2}(x)-u_{1}(x)\right)$. Then $\eta_{i}$ is a Lipschitz continuous function on $\Omega$ which vanishes on a neighborhood of $\Gamma$ (here we use the fact that $u_{1}$ and $u_{2}$ are continuous up to the boundary and agree on the boundary). Hence, we can insert $\eta_{i}$ in (E), which yields:

$$
\int_{\Omega}\left\langle a\left(\nabla u_{1}\right), \nabla \eta_{i}\right\rangle-F\left[u_{1}\right] \eta_{i}=0 .
$$

Since $\nabla \eta_{i}=\theta^{\prime}\left(i\left(u_{2}-u_{1}\right)\right)\left(\nabla u_{2}-\nabla u_{1}\right)$, we get

$$
\int_{\Omega} \theta^{\prime}\left(i\left(u_{2}-u_{1}\right)\right)\left\langle a\left(\nabla u_{1}\right), \nabla u_{2}-\nabla u_{1}\right\rangle-F\left[u_{1}\right] \eta_{i}=0 .
$$

Symetrically, we have (with $u_{2},-\eta_{i}$ instead of $u_{1}, \eta_{i}$ )

$$
\int_{\Omega} \theta^{\prime}\left(i\left(u_{1}-u_{2}\right)\right)\left\langle a\left(\nabla u_{2}\right), \nabla u_{1}-\nabla u_{2}\right\rangle+F\left[u_{2}\right] \eta_{i}=0 .
$$

The sum of (3.14) and (3.15) gives

$$
\int_{\Omega} \theta^{\prime}\left(i\left(u_{2}-u_{1}\right)\right)\left\langle a\left(\nabla u_{2}\right)-a\left(\nabla u_{1}\right), \nabla u_{2}-\nabla u_{1}\right\rangle=\int_{\Omega}\left(F\left[u_{1}\right]-F\left[u_{2}\right]\right) \frac{\theta\left(i\left(u_{1}-u_{2}\right)\right)}{i} .
$$

Here, we have used the fact that $\theta(x)=-\theta(-x)$ and $\theta^{\prime}(x)=\theta^{\prime}(-x)$. Using (1.8), we get

$$
\mu \int_{\Omega} \theta^{\prime}\left(i\left(u_{2}-u_{1}\right)\right)\left|\nabla u_{2}-\nabla u_{1}\right|^{2} \mathrm{~d} x \leq \int_{\Omega}\left(F\left[u_{1}\right]-F\left[u_{2}\right]\right) \frac{\theta\left(i\left(u_{1}-u_{2}\right)\right)}{i} .
$$

By the dominated convergence theorem, the right hand side goes to $\int_{\Omega}\left(F\left[u_{1}\right]-F\left[u_{2}\right]\right)\left(u_{1}-u_{2}\right)$ when $i \rightarrow \infty$. This quantity is nonpositive by (3.13). Hence, by Fatou's Lemma in the left hand side, we have

$$
\liminf _{i \rightarrow \infty} \theta^{\prime}\left(i\left(u_{2}-u_{1}\right)(x)\right)\left|\nabla u_{2}(x)-\nabla u_{1}(x)\right|^{2}=0, \quad \text { a.e. } x \in \Omega .
$$

This implies that

$$
\left(u_{2}-u_{1}\right)(x)\left|\nabla u_{2}(x)-\nabla u_{1}(x)\right|^{2}=0, \quad \text { a.e. } x \in \Omega
$$

so that $u_{1}=u_{2}$ on $\Omega$. This completes the proof of Theorem 2.4. 


\section{Proof of Theorem 2.5}

Exactly as in the proof of Theorem 2.3, for each $K \geq K_{\phi}$, there exists a $K$ quasi solution $u_{K} \in \operatorname{Lip}_{\phi}(\Omega, K)$. There exists $T>0$ such that for any $K \geq K_{\phi}$,

$$
\left\|u_{K}\right\|_{L^{\infty}(\Omega)} \leq T, \quad\left\|F\left[u_{K}\right]\right\|_{L^{\infty}(\Omega)} \leq \chi(T) .
$$

We observe that for any $\xi, \xi^{\prime} \in \mathbb{R}^{n}$,

$$
\left\langle a(\xi)-a\left(\xi^{\prime}\right), \xi-\xi^{\prime}\right\rangle \geq \frac{\mu}{2^{p}}\left|\xi-\xi^{\prime}\right|^{p}
$$

Indeed, let $r=|\xi|, s=\left|\xi^{\prime}\right|$ and $b=\left\langle\xi /|\xi|, \xi^{\prime} /\left|\xi^{\prime}\right|\right\rangle$. Then

$$
\left\langle a(\xi)-a\left(\xi^{\prime}\right), \xi-\xi^{\prime}\right\rangle=r l(r)+s l(s)-b(s l(r)+r l(s))
$$

and $\left|\xi-\xi^{\prime}\right|^{p}=\left(r^{2}+s^{2}-2 b r s\right)^{p / 2}$. Then (4.2) is equivalent to

$$
r l(r)+s l(s)-b(s l(r)+r l(s)) \geq \frac{\mu}{2^{p}}\left(r^{2}+s^{2}-2 b r s\right)^{p / 2}, \quad r, s \geq 0,-1 \leq b \leq 1 .
$$

By convexity of the right hand side with respect to $b$, we only need to prove (4.3) when $b \in\{-1,1\}$. When $b=1$, this amounts to

$$
(l(r)-l(s))(r-s) \geq \frac{\mu}{2^{p}}|r-s|^{p} .
$$

This follows from $(H l)$ at once. When $b=-1$, we have to prove that

$$
(l(r)+l(s))(r+s) \geq \frac{\mu}{2^{p}}(r+s)^{p} .
$$

By $(H l)$ and the fact that $l(0)=0$, we have $l(r) \geq \mu r^{p-1}, l(s) \geq \mu s^{p-1}$. This implies (4.4) and thus (4.3) is also true in that case. This completes the proof of (4.2).

Step 1: A variational setting. We proceed to prove that $u_{K}$ is the solution of a variational problem on $\operatorname{Lip}_{\phi}(\Omega, K)$. We introduce $\lambda(t):=\int_{0}^{t} l(s) \mathrm{d} s$ and $L(\xi):=\lambda(|\xi|)$. Then $L$ is non negative, convex and differentiable with $\nabla L(\xi)=l(|\xi|) \xi /|\xi|=a(\xi)$.

Let $K \geq K_{\phi}$ and $v \in \operatorname{Lip}_{\phi}(\Omega, K)$. For any $x \in \Omega$, the function

$$
t \mapsto L\left(\nabla u_{K}(x)+t \nabla\left(v-u_{K}\right)(x)\right)
$$

is convex. Hence, the function

$$
g: t \mapsto \int_{\Omega} L\left(\nabla u_{K}+t\left(\nabla v-\nabla u_{K}\right)\right)-F\left[u_{K}\right]\left(u_{K}+t\left(v-u_{K}\right)\right)
$$

is convex as well. Since $v$ and $u_{K}$ are Lipschitz continuous, their gradients are uniformly bounded and we can differentiate under the integral sign. We have

$$
g^{\prime}(0)=\int_{\Omega}\left\langle a\left(\nabla u_{K}\right), \nabla v-\nabla u_{K}\right\rangle-F\left[u_{K}\right]\left(v-u_{K}\right) .
$$

Since $u_{K}$ is a $K$ quasi solution, we get $g^{\prime}(0) \geq 0$ so that $g$ is non decreasing on $[0,+\infty)$. Whence

$$
\int_{\Omega} L\left(\nabla u_{K}\right)-F\left[u_{K}\right] u_{K} \leq \int_{\Omega} L(\nabla v)-F\left[u_{K}\right] v
$$


By $(H l), L\left(\nabla u_{K}\right) \geq \frac{\mu}{p}\left|\nabla u_{K}\right|^{p}$. By (4.5) with $v=\phi$ and (4.1), there exists $S>0$ (independent of $K$ ) such that $\left\|u_{K}\right\|_{W^{1, p}(\Omega)} \leq S$.

Step 2: A uniform Hölder continuity estimate for quasi solutions. In order to establish a Hölder estimate, we need a generalization of Lemma 3.8:

Lemma 4.1. Assume that $a \in C^{0}\left(\mathbb{R}^{n}, \mathbb{R}^{n}\right)$ satisfies

$$
\left\langle a(\xi)-a\left(\xi^{\prime}\right), \xi-\xi^{\prime}\right\rangle \geq \nu\left|\xi-\xi^{\prime}\right|^{p}, \quad \xi, \xi^{\prime} \in \mathbb{R}^{n}
$$

for some $\nu>0, p>1$. Let $\phi: \mathbb{R}^{n} \rightarrow \mathbb{R}$ be a Lipschitz map of rank $K_{\phi}, g \in L^{\infty}(\Omega)$ and $K \geq K_{\phi}$. Let $u \in \operatorname{Lip}_{\phi}(\Omega, K)$ such that

$$
\int_{\Omega}\langle a(\nabla u), \nabla v-\nabla u\rangle-g(x)(v-u) \geq 0, \quad v \in \operatorname{Lip}_{\phi}(\Omega, K) .
$$

Then there exists $C_{0}>0$ depending only on $\mu /\|g\|_{L^{\infty}(\Omega)}$ and $|\Omega|$ such that

$$
|u(x)-u(y)| \leq \max _{\substack{z \in \Omega, \gamma \in \Gamma \\|z-\gamma| \leq|x-y|}}|u(z)-\phi(\gamma)|+C_{0}|x-y|^{\frac{1}{p-1}} .
$$

Proof. This is a mere adaptation of [8] Lemma 10.0. Fix $\bar{x}, \bar{y}$ in $\Omega$ and set $\tau:=\bar{y}-\bar{x}$. We define $u_{\tau}(\cdot)=u(\cdot+\tau)$ on $\Omega_{\tau}:=\Omega-\tau$, as well as $\phi_{\tau}(\cdot)=\phi(\cdot+\tau)$ and $g_{\tau}(\cdot)=g(\cdot+\tau)$. Then

$$
\int_{\Omega_{\tau}}\left\langle a\left(\nabla u_{\tau}\right), \nabla w-\nabla u_{\tau}\right\rangle-g_{\tau}(x)\left(w-u_{\tau}\right) \geq 0, \quad w \in \operatorname{Lip}_{\phi_{\tau}}\left(\Omega_{\tau}, K\right) .
$$

Let $M \geq M_{\tau}:=\max _{z \in \Omega, \gamma \in \Gamma}|u(z)-\phi(\gamma)|$. We observe that for any $x \in \partial\left(\Omega \cap \Omega_{\tau}\right), x \in \partial \Omega$ or $x+\tau \in \partial \Omega$. Hence, $\left|u(x)-u_{\tau}(x)\right| \begin{gathered}|z-\gamma| \leq \tau \\ \leq M\end{gathered}$ on $\partial\left(\Omega \cap \Omega_{\tau}\right)$.

In (4.7), we take

$$
v:=\left\{\begin{array}{l}
u \text { on } \Omega \backslash \Omega_{\tau}, \\
\max \left(u, u_{\tau}-M\right) \text { on } \Omega \cap \Omega_{\tau} .
\end{array}\right.
$$

We get

$$
\int_{A_{M}}\left\langle a(\nabla u), \nabla u_{\tau}-\nabla u\right\rangle-g(x)\left(u_{\tau}-u-M\right) \geq 0
$$

where $A_{M}:=\left\{x \in \Omega \cap \Omega_{\tau}: u(x) \leq u_{\tau}(x)-M\right\}$. Now, we define

$$
w:=\left\{\begin{array}{l}
u_{\tau} \text { on } \Omega_{\tau} \backslash \Omega, \\
\min \left(u+M, u_{\tau}\right) \text { on } \Omega \cap \Omega_{\tau} .
\end{array}\right.
$$

By inserting $w$ in (4.9), we get

$$
\int_{A_{M}}\left\langle a\left(\nabla u_{\tau}\right), \nabla u-\nabla u_{\tau}\right\rangle-g_{\tau}(x)\left(u-u_{\tau}+M\right) \geq 0 .
$$

By adding (4.10) and (4.11) and in view of (4.6), we get

$$
\nu \int_{A_{M}}\left|\nabla u_{\tau}-\nabla u\right|^{p} \leq \int_{A_{M}}\left(g_{\tau}(x)-g(x)\right) v(x) \mathrm{d} x
$$


where $v(x):=\max \left(u_{\tau}(x)-u(x)-M, 0\right) \in \operatorname{Lip}_{0}\left(\Omega \cap \Omega_{\tau}\right)$. We extend $g$ and $v$ on $\mathbb{R}^{n}$ by 0 . The right hand side of (4.12) is equal to

$$
\int_{\mathbb{R}^{n}} g(x)(v(x-\tau)-v(x)) \mathrm{d} x \leq\|g\|_{L^{\infty}(\Omega)}|\tau| \int_{\mathbb{R}^{n}}|\nabla v(x)| \mathrm{d} x .
$$

By (4.12) and (4.13), we have

$$
\int_{A_{M}}\left|\nabla u-\nabla u_{\tau}\right|^{p} \leq \frac{\|g\|_{L^{\infty}(\Omega)}}{\nu}|\tau| \int_{A_{M}}\left|\nabla u-\nabla u_{\tau}\right| .
$$

By Hölder inequality, this gives

$$
\left(\int_{A_{M}}\left|\nabla u-\nabla u_{\tau}\right|^{p}\right)^{\frac{1}{p}} \leq\left(\frac{\|g\|_{L^{\infty}(\Omega)}}{\nu}|\tau|\right)^{\frac{1}{p-1}}\left|A_{M}\right|^{\frac{1}{p}} .
$$

Hence, by Fubini Theorem and the definition of $A_{M}, M \geq M_{\tau}$, we obtain

$$
\begin{aligned}
\int_{M}^{+\infty}\left|A_{M^{\prime}}\right| \mathrm{d} M^{\prime} & =\int_{A_{M}}\left(u_{\tau}(x)-u(x)-M\right) \mathrm{d} x \\
& \leq\left(\int_{A_{M}}\left|u_{\tau}-u-M\right|^{p^{*}}\right)^{\frac{1}{p^{*}}}\left|A_{M}\right|^{1-\frac{1}{p^{*}}} \leq C\left(\int_{A_{M}}\left|\nabla u_{\tau}-\nabla u\right|^{p}\right)^{\frac{1}{p}}\left|A_{M}\right|^{1-\frac{1}{p^{*}}} \\
& \leq C^{\prime}|\tau|^{\frac{1}{p-1}}\left|A_{M}\right|^{\beta},
\end{aligned}
$$

where $\beta>1$ while $C$ and $C^{\prime}$ depend only on $n, p, \Omega$ and $\|g\|_{L^{\infty}(\Omega)} / \nu$. Here, we have used the Sobolev inequality with $p^{*}=n p /(n-p)$ if $p<n$ (in that case, $\left.\beta=1+1 / n\right)$. When $p \geq n$, we take for $p^{*}$ any exponent larger than $p$.

This implies (see [8] Lem. 7.2) that $\left|A_{M}\right|=0$ for

$$
M \geq C^{\prime} \frac{\beta}{\beta-1}|\tau|^{\frac{1}{p-1}}|\Omega|^{\beta-1}+M_{\tau} .
$$

In particular, $u_{\tau}(\bar{x}) \leq u(\bar{x})+C_{0}|\tau|^{1 /(p-1)}+M_{\tau}\left(C_{0}=C^{\prime}|\Omega|^{\beta-1} \beta /(\beta-1)\right)$. The other inequality can be established similarly. Since $\tau=\bar{y}-\bar{x}$, this completes the proof of Lemma 4.1.

We can now state the Hölder estimate for $K$ quasi solutions:

Proposition 4.2. There exists $C>0$ such that for any $K \geq K_{\phi}$, for any $K$ quasi solution $u_{K}$,

$$
\left|u_{K}(x)-u_{K}(y)\right| \leq C|x-y|^{\alpha} \quad \forall x, y \in \Omega
$$

where $\alpha:=\min \left(\frac{p-1}{n+p-1}, \frac{1}{p-1}\right)$.

Proof. By Lemma 4.1, it is enough to prove that there exists a positive constant $C$ such that for any $\gamma \in \Gamma$, for any $K \geq K_{\phi}$ and any $K$ quasi solution $u$ we have

$$
|u(x)-u(\gamma)| \leq C|x-\gamma|^{\frac{p-1}{n+p-1}}, \quad \forall x \in \Omega .
$$

We proceed to prove that $u(x)-u(\gamma) \leq C|x-\gamma|^{(p-1) /(n+p-1)}$. The other inequality could be established similarly. 
Fix $\gamma \in \Gamma$. There exists $r>0$ not depending on $\gamma$ and $z \in \mathbb{R}^{n}$ such that $B(z, r) \subset \mathbb{R}^{n} \backslash \Omega$ and $|z-\gamma|=r$. Let $R:=r+\operatorname{diam} \Omega$. We define $\Omega^{*}:=B(z, R) \backslash \bar{B}(z, r)$ and

$$
\phi^{*}(x):=\phi(\gamma)+K_{\phi}|x-\gamma| .
$$

Fix $K \geq K_{\phi}$ and consider $u$ a $K$ quasi solution of $(E)$.

There exists a $K$ quasi solution solution $v \in \operatorname{Lip}_{\phi^{*}}\left(\Omega^{*}, K\right)$ to the inequation

$$
\int_{\Omega^{*}}\langle a(\nabla v), \nabla w-\nabla v\rangle-\chi(T)(w-v) \geq 0, \quad \forall w \in \operatorname{Lip}_{\phi^{*}}\left(\Omega^{*}, K\right) .
$$

Moreover, there exist $T^{*}>0$ and $S^{*}>0$ independent of $K$ such that

$$
\|v\|_{L^{\infty}\left(\Omega^{*}\right)} \leq T^{*},\|v\|_{W^{1, p}\left(\Omega^{*}\right)} \leq S^{*} .
$$

By Lemma 3.6 (wih $u^{*}=v$ ), we get $v \geq u$ on $\Omega$.

We claim that the Hölder norm of $v$ can be estimated independently of $K$ and $\gamma$. The proof is very similar to the proof of Lemma 4.1 except that we replace translations by rotations. This is the main reason why we require that $a$ be radial. Without loss of generality, we can assume that $z=0$. For any linear isometry $I: \mathbb{R}^{n} \rightarrow \mathbb{R}^{n}$, $I\left(\Omega^{*}\right)=\Omega^{*}$. For any $w \in \operatorname{Lip}_{\phi^{*}}\left(\Omega^{*}\right)$, we denote by $w_{I}$ the map $w \circ I$. By (4.16) and an obvious change of variables, we have

$$
\int_{\Omega^{*}}\left\langle a\left(\nabla v_{I}\right), \nabla w_{I}-\nabla v_{I}\right\rangle-\chi(T)\left(w_{I}-v_{I}\right) \geq 0, \quad \forall w \in \operatorname{Lip}_{\phi^{*}}\left(\Omega^{*}, K\right) .
$$

Here, we have also used the fact that

$$
\begin{aligned}
\left\langle a\left(\nabla v_{I}\right), \nabla w_{I}-\nabla v_{I}\right\rangle & =\left\langle a\left(I^{*}(\nabla v \circ I)\right), I^{*}(\nabla w \circ I-\nabla v \circ I)\right\rangle \\
& =\frac{l(|\nabla v \circ I|)}{|\nabla v \circ I|}\left\langle I^{*}(\nabla v \circ I), I^{*}(\nabla w \circ I-\nabla v \circ I)\right\rangle \\
& =\langle a(\nabla v \circ I), \nabla w \circ I-\nabla v \circ I\rangle .
\end{aligned}
$$

Let $b:=\max _{|y|=1}|I(y)-y|$. We now take $w=\max \left(v_{I}-K_{\phi} R b, v\right) \in \operatorname{Lip}_{\phi^{*}}\left(\Omega^{*}, K\right)$ in (4.16):

$$
\int_{\left[v_{I}-K_{\phi} R b>v\right]}\left\langle a(\nabla v), \nabla v_{I}-\nabla v\right\rangle-\chi(T)\left(v_{I}-K_{\phi} R b-v\right) \geq 0 .
$$

With $w=\min \left(v, v \circ I^{-1}+K_{\phi} R b\right) \in \operatorname{Lip}_{\phi^{*}}\left(\Omega^{*}, K\right)$ in (4.17), we have:

$$
\int_{\left[v_{I}-K_{\phi} R b>v\right]}\left\langle a\left(\nabla v_{I}\right), \nabla v-\nabla v_{I}\right\rangle+\chi(T)\left(v_{I}-K_{\phi} R b-v\right) \geq 0 .
$$

The sum of (4.18) and (4.19) (see also (4.2)) leads to

$$
\int_{\left[v_{I}-K_{\phi} R b>v\right]}\left|\nabla v-\nabla v_{I}\right|^{p} \leq 0 .
$$

Hence, for any $x \in \Omega^{*}, v_{I}(x)-v(x) \leq K_{\phi} R b$. Symetrically, we have $v \leq v_{I}+K_{\phi} R b$.

Now, if $x, y \in \Omega^{*}$ are such that $|x|=|y|$, there exists an isometry $I$ such that $I(x)=y$ and

$$
|v(x)-v(y)|=\left|v(x)-v_{I}(x)\right| \leq K_{\phi} R b \leq Q|x-y|
$$


for some constant $Q$ which depends only on $K_{\phi}, r$ and $R$. We now apply to $v$ the following lemma which is a 'spherical' version of Lemma 3.5:

Lemma 4.3. Let $v \in W^{1, p}(B(0, R) \backslash \overline{B(0, r)})$. Assume that there exists $Q>0$ such that for any $x, y \in B(0, R) \backslash$ $\overline{B(0, r)},|x|=|y|$, we have

$$
|v(x)-v(y)| \leq Q|x-y| .
$$

Then there exists $C>0$ depending only on $Q,\|v\|_{W^{1, p}(B(0, R) \backslash \overline{B(0, r)})}, r$ and $R$ such that for any $x, y \in B(0, R) \backslash$ $\overline{B(0, r)}$,

$$
|v(x)-v(y)| \leq C|x-y|^{\frac{p-1}{n+p-1}} .
$$

Lemma 4.3 easily follows from Lemma 3.5 by the change of variables formula (see [3], Lem. 5 for a detailed proof). It gives an estimate of the Hölder norm of the quasi solution $v$ of (4.16). Since $u \leq v$ on $\Omega$ and $u(\gamma)=\phi(\gamma)$ $=\phi^{*}(\gamma)=v(\gamma)$, we thus get

$$
u(x)-u(\gamma) \leq C|x-\gamma|^{\frac{p-1}{n+p-1}}, \quad x \in \Omega,
$$

where $C$ depends neither on $\gamma$ nor on $K$. This completes the proof of Proposition 4.2.

By Proposition 4.2, a subsequence of quasi solutions $\left(u_{K_{i}}\right)$ uniformly converges to a Hölder continuous function $u$ satisfying (4.14). Since $\left(u_{K_{i}}\right)$ is bounded in $W^{1, p}(\Omega)$, we can further assume that $\left(u_{K_{i}}\right)$ weakly converges to $u$ in $W^{1, p}(\Omega)$.

The convexity of $L$ implies that $\int_{\Omega} L(\nabla u) \leq \liminf _{i \rightarrow+\infty} \int_{\Omega} L\left(\nabla u_{K_{i}}\right)$. From (4.5) and (HF1), we thus get for any $v \in \operatorname{Lip}_{\phi}(\Omega)$

$$
\int_{\Omega} L(\nabla u)-F[u] u \leq \int_{\Omega} L(\nabla v)-F[u] v .
$$

In particular, $L(\nabla u) \in L^{1}(\Omega)$. We proceed to prove that $u$ is a solution of $(E)$. The map $u$ is not Lipschitz continuous so that we cannot differentiate under the integral sign. Still, the minimum of a problem in the calculus of variations is a solution of the corresponding Euler equation when the Lagrangian $L$ is convex (and does not depend on $x$ and $u$ ). This result recently proved in [5] does not require any growth assumption on $L$. We cannot directly apply it because in our situation, the admissible maps are Lipschitz continuous whereas $u$ only belongs to $W^{1, p}(\Omega)$. We have to prove somehow that no Lavrentiev phenomenon can occur.

Step 3: An approximation Lemma. We first state

Lemma 4.4. There exists a sequence $\left(u_{k}\right)_{k \geq 1} \subset W_{\phi}^{1,1}(\Omega)$ converging to $u$ in $W^{1,1}(\Omega)$ such that

(1) for each $k \geq 1, u_{k}$ is Lipschitz continuous on a neighborhood of $\partial \Omega$ in $\Omega$;

(2) for each $k \geq 1, L\left(\nabla u_{k}\right) \in L^{1}(\Omega)$;

(3) $\left(L\left(\nabla u_{k}\right)\right)_{k \geq 1}$ converges to $L(\nabla u)$ in $L^{1}(\Omega)$.

Proof of Lemma 4.4. Since $\Gamma$ is locally the graph of Lipschitz maps, there exist $\delta>0$, and finitely many cubes $Q\left(x_{i}, \delta\right)$ of centers $x_{i}$ and radius $\delta>0, i=1, \ldots, M$, such that $\Gamma \subset \cup_{i=1}^{M} Q\left(x_{i}, \delta / 4\right)$ and for each $i$, there is an isometry $\zeta_{i}$ of $\mathbb{R}^{n}$ which maps $Q\left(x_{i}, \delta\right)$ onto $(-\delta, \delta)^{n}$ and $Q\left(x_{i}, \delta\right) \cap \Omega$ onto

$$
U_{i}:=\left\{\left(x^{\prime}, x_{n}\right) \in(-\delta, \delta)^{n-1} \times(\delta, \delta): x_{n}>h_{i}\left(x^{\prime}\right)\right\},
$$

where $h_{i}:[-\delta, \delta]^{n-1} \rightarrow \mathbb{R}$ is Lipschitz continuous. We can further assume that

$$
\zeta_{i}\left(\partial \Omega \cap Q\left(x_{i}, \delta\right)\right)=\left\{\left(y^{\prime}, h_{i}\left(y^{\prime}\right)\right): y^{\prime} \in(-\delta, \delta)^{n-1}\right\} .
$$


Let $\theta \in C_{c}^{\infty}\left((-\delta, \delta)^{n}\right)$ be such that $0 \leq \theta \leq 1$ and $\theta=1$ on $(-\delta / 2, \delta / 2)^{n}$. We introduce

$$
\psi_{k}(x):=x-\frac{1}{k} \theta(x) e_{n}
$$

where $e_{n}:=(0, \ldots, 0,1)$. Clearly, for any $k \geq 1$,

$$
\left\|\psi_{k}-I d\right\|_{L^{\infty}}+\left\|D \psi_{k}-I d\right\|_{L^{\infty}} \leq \frac{C}{k}
$$

Moreover, $\psi_{k}=I d$ outside $(-\delta, \delta)^{n}$. We claim that there exists $C^{\prime}>0$ such that for any $k \geq 1$ and for any $i=1, \ldots, M$, we have

$$
\begin{aligned}
& \mathrm{d}\left(\psi_{k}(x), \overline{U_{i}}\right) \geq \frac{C^{\prime}}{k} \text { when } x \in(-\delta / 2, \delta / 2)^{n} \backslash U_{i}, \\
& \mathrm{~d}\left(\psi_{k}(x), \overline{U_{i}}\right) \geq C^{\prime} \mathrm{d}\left(x, \overline{U_{i}}\right) \text { when } x \in(-\delta, \delta)^{n} \backslash U_{i} .
\end{aligned}
$$

Indeed, let $z=\left(z^{\prime}, z_{n}\right) \in(-\delta, \delta)^{n} \backslash U_{i}$. We then consider $y=\left(y^{\prime}, h\left(y^{\prime}\right)\right) \in \overline{U_{i}}$ such that $|z-y|=\mathrm{d}\left(z, \overline{U_{i}}\right)$. Then there exists $B>0$ such that

$$
h_{i}\left(z^{\prime}\right)-z_{n} \leq\left|z_{n}-h_{i}\left(y^{\prime}\right)\right|+\left|h_{i}\left(y^{\prime}\right)-h_{i}\left(z^{\prime}\right)\right| \leq B|z-y|=B \mathrm{~d}\left(z, \overline{U_{i}}\right) .
$$

If $z=\psi_{k}(x)$ for some $x \in(-\delta / 2, \delta / 2)^{n} \backslash U_{i}$, then $z^{\prime}=x^{\prime}$ and

$$
\frac{1}{k}=|x-z| \leq h_{i}\left(x^{\prime}\right)-z_{n}=h_{i}\left(z^{\prime}\right)-z_{n} \leq B \mathrm{~d}\left(z, \overline{U_{i}}\right) .
$$

This implies (4.24) while (4.25) can be proved similarly.

We now introduce $\psi_{k}^{i}=\zeta_{i}^{-1} \circ \psi_{k} \circ \zeta_{i}$. Then $\psi_{k}^{i}$ satisfies $(4.23)-(4.25)$ in $Q\left(x_{i}, \delta\right)$. We extend $\psi_{k}^{i}$ by the identity outside $Q\left(x_{i}, \delta\right)$. Finally, we define

$$
\Phi_{k}:=\psi_{k}^{1} \circ \cdots \circ \psi_{k}^{M} .
$$

We easily get from (4.23)-(4.25), that the map $\Phi_{k}$ satisfies

$$
\begin{array}{r}
\left\|\Phi_{k}-I d\right\|_{L^{\infty}}+\left\|D \Phi_{k}-I d\right\|_{L^{\infty}} \leq \frac{A_{0}}{k}, \\
\mathrm{~d}\left(\Phi_{k}(\Gamma), \bar{\Omega}\right) \geq \frac{A}{k}
\end{array}
$$

for some positive $A_{0}, A>0$. By relabelling the sequence $\Phi_{k}$, we can assume that $A_{0}=1$.

There exists a constant $C_{0}>0$ such that for any $k \geq 1$,

$$
\left\|D \Phi_{k}\right\|_{L^{\infty}}+\left\|D\left(\Phi_{k}\right)^{-1}\right\|_{L^{\infty}} \leq C_{0} .
$$

For each $k \geq 1$, we define on $\Gamma \cup \Phi_{k}(\Gamma)$

$$
\tilde{\phi}_{k}: y \mapsto\left\{\begin{array}{l}
\phi(y) \text { on } \Gamma \\
\left\|D \Phi_{k}\right\|_{L^{\infty}} \phi\left(\Phi_{k}^{-1}(y)\right) \text { on } \Phi_{k}(\Gamma) .
\end{array}\right.
$$

We claim that $\tilde{\phi}_{k}$ is Lipschitz continuous of rank not larger than

$$
\bar{C}:=\max \left(K_{\phi}, C_{0}^{2} K_{\phi}, K_{\phi}(1+1 / A)+\|\phi\|_{L^{\infty}} / A\right) .
$$


Indeed, we only need to prove that for any $y, z \in \Gamma$, we have $\left|\tilde{\phi}_{k}(y)-\tilde{\phi}_{k}\left(\Phi_{k}(z)\right)\right| \leq \bar{C}\left|y-\Phi_{k}(z)\right|$. We have

$$
\begin{aligned}
\left|\tilde{\phi}_{k}(y)-\tilde{\phi}_{k}\left(\Phi_{k}(z)\right)\right| & =|\phi(y)-|\left|D \Phi_{k} \|_{L^{\infty}} \phi(z)\right| \\
& \leq|\phi(y)-\phi(z)|+|\phi(z)-|\left|D \Phi_{k}\left\|_{L^{\infty}} \phi(z)\left|\leq K_{\phi}\right| y-z\left|+\|\phi\|_{L^{\infty}}\right| 1-\right\| D \Phi_{k} \|_{L^{\infty}}\right| \\
& \leq K_{\phi}\left|y-\Phi_{k}(z)\right|+K_{\phi}\left|\Phi_{k}(z)-z\right|+\|\phi\|_{L^{\infty}}|| I d-D \Phi_{k} \|_{L^{\infty}} \\
& \leq K_{\phi}\left|y-\Phi_{k}(z)\right|+\frac{K_{\phi}+\|\left.\phi\right|_{L^{\infty}}}{k}
\end{aligned}
$$

by using (4.26). By (4.27), we get

$$
\begin{aligned}
\left|\tilde{\phi}_{k}(y)-\tilde{\phi}_{k}\left(\Phi_{k}(z)\right)\right| & \leq K_{\phi}\left|y-\Phi_{k}(z)\right|+\frac{1}{A}\left(K_{\phi}+\|\phi\|_{L^{\infty}}\right) \mathrm{d}\left(\Gamma, \Phi_{k}(\Gamma)\right) \\
& \leq\left(K_{\phi}\left(1+\frac{1}{A}\right)+\frac{1}{A}|| \phi \|_{L^{\infty}}\right)\left|y-\Phi_{k}(z)\right| .
\end{aligned}
$$

The claim is proved.

We still denote by $\tilde{\phi}_{k}$ a Lipschitz extension on $\mathbb{R}^{n}$ of $\tilde{\phi}_{k}$ with Lipschitz rank not larger than $\bar{C}$. We then introduce

$$
\tilde{u}_{k}(x):=\left\{\begin{array}{l}
u(x) \text { if } x \in \Omega \\
\tilde{\phi}_{k}(x) \text { if } x \in \mathbb{R}^{n} \backslash \Omega .
\end{array}\right.
$$

Finally, we define for $x \in \Omega$

$$
u_{k}(x):=\frac{1}{\left\|D \Phi_{k}(x)\right\|_{L^{\infty}}} \tilde{u}_{k} \circ \Phi_{k}(x) .
$$

Since $\Phi_{k}$ converges to $I d$ in the $C^{1}$ topology, $\left(u_{k}\right)$ converges to $u$ in $W^{1,1}(\Omega)$. There exists a subsequence that we still denote by $\left(u_{k}\right)$ such that $\left(\nabla u_{k}\right)$ converges to $\nabla u$ almost everywhere in $\Omega$.

Moreover, for any $\gamma \in \Gamma$, we have

$$
u_{k}(\gamma)=\frac{1}{\left\|D \Phi_{k}\right\|_{L^{\infty}}} \tilde{u}_{k} \circ \Phi_{k}(\gamma)=\frac{1}{\left\|D \Phi_{k}\right\|_{L^{\infty}}} \tilde{\phi}_{k}\left(\Phi_{k}(\gamma)\right)=\phi(\gamma) .
$$

For each $k \geq 1$, there exists $\delta_{k}>0$ such that for any $x \in \Omega$ satisfying $\mathrm{d}(x, \Gamma)<\delta_{k}$, we have $\Phi_{k}(x) \notin \Omega$.

Since $u_{k}(x)=\frac{1}{\left\|D \Phi_{k}\right\|_{L^{\infty}}} \tilde{\phi}_{k} \circ \Phi_{k}(x)$ when $\mathrm{d}(x, \Gamma)<\delta_{k}$, the map $u_{k}$ is Lipschitz continuous on a neighborhood of $\Gamma$ in $\Omega$.

We claim that

$$
\limsup _{k \rightarrow \infty} \int_{\Omega} L\left(\nabla u_{k}\right) \leq \int_{\Omega} L(\nabla u)
$$

This follows from the fact that $\lambda$ is non decreasing:

$$
\begin{aligned}
\int_{\Omega} L\left(\nabla u_{k}\right) & =\int_{\Omega} \lambda\left(\mid \frac{1}{\left\|D \Phi_{k}\right\|_{\infty}} D \Phi_{k}(x)^{*}\left(\nabla \tilde{u}_{k}\left(\Phi_{k}(x)\right) \mid\right) \mathrm{d} x\right. \\
& \leq \int_{\Omega \cap \Phi_{k}^{-1}(\Omega)} \lambda\left(\left|\nabla u\left(\Phi_{k}(x)\right)\right|\right) \mathrm{d} x+\lambda(\bar{C})\left|\Omega \backslash \Phi_{k}^{-1}(\Omega)\right| .
\end{aligned}
$$

By the change of variables formula, we then get

$$
\int_{\Omega} L\left(\nabla u_{k}\right) \leq \int_{\Omega} L(\nabla u(y))\left|J a c \Phi_{k}^{-1}(y)\right| \mathrm{d} y+\lambda(\bar{C})\left|\Omega \backslash \Phi_{k}^{-1}(\Omega)\right| .
$$


By the dominated convergence theorem, the right hand side converges to $\int_{\Omega} L(\nabla u)$. This implies inequality (4.28). By Fatou Lemma,

$$
\liminf _{k \rightarrow+\infty} \int_{\Omega} L\left(\nabla u_{k}\right) \geq \int_{\Omega} L(\nabla u) .
$$

Hence, $\left(L\left(\nabla u_{k}\right)\right)_{k \geq 1}$ converges to $L(\nabla u)$ in $L^{1}(\Omega)$. This completes the proof of Lemma 4.4.

Finally, we have the following approximation lemma:

Lemma 4.5. There exists $\left(u_{m}\right) \subset \operatorname{Lip}_{\phi}(\Omega)$ such that $\left(u_{m}\right)$ converges to $u$ in $W^{1,1}(\Omega)$ and $\left(L\left(\nabla u_{m}\right)\right)$ converges to $L(\nabla u)$ in $L^{1}(\Omega)$.

Proof. By Lemma 4.4, we can assume that $u$ is Lipschitz continuous near the boundary. We extend $u$ by a Lipschitz continuous function outside $\Omega$. Let $K$ be a compact subset of $\Omega$ such that $u$ is Lipschitz continuous on $\bar{\Omega} \backslash K$. Let $K_{1}$ be a compact subset of $\Omega$ such that int $K_{1} \supset K$. We introduce $\theta \in C_{c}^{\infty}(\Omega)$ such that $0 \leq \theta \leq 1$, $\theta=1$ on $K_{1}$. Let $\rho \in C_{c}^{\infty}(B(0,1)), \rho \geq 0, \int_{\mathbb{R}^{n}} \rho=1$ and $\rho_{k}(\cdot):=k^{n} \rho(k \cdot)$. We then define

$$
u_{k}(x):=\theta(x)\left(u * \rho_{k}\right)(x)+(1-\theta(x)) u(x) .
$$

Then $u_{k}$ is Lipschitz continuous on $\bar{\Omega}$. We have

$$
\nabla u_{k}(x)=\theta(x)\left(\nabla u * \rho_{k}\right)(x)+(1-\theta(x)) \nabla u(x)+\left(u * \rho_{k}(x)-u(x)\right) \nabla \theta(x) .
$$

This implies that $\left(u_{k}\right)$ converges in $W^{1,1}(\Omega)$ to $u$ and (up to a subsequence), $\left(\nabla u_{k}\right)$ converges to $\nabla u$ a.e. We also observe that when $x \in K_{1}, \nabla u_{k}(x)=\left(\nabla u * \rho_{k}\right)(x)$. For $k>1 / \mathrm{d}\left(K, \partial K_{1}\right),\left\|\nabla u * \rho_{k}\right\|_{L^{\infty}\left(\Omega \backslash K_{1}\right)} \leq\|\nabla u\|_{L^{\infty}\left(\mathbb{R}^{n} \backslash K\right)}$. Hence, by considering the two cases $x \in K_{1}$ and $x \notin K_{1}$ separately, we get for any $k>1 / \mathrm{d}\left(K, \partial K_{1}\right)$

$$
L\left(\nabla u_{k}(x)\right) \leq K_{L}|| \nabla \theta \|_{L^{\infty}(\Omega)}\left|u * \rho_{k}(x)-u(x)\right|+L\left(\theta(x)\left(\nabla u * \rho_{k}\right)(x)+(1-\theta(x)) \nabla u(x)\right)
$$

where $K_{L}$ is a Lipschitz rank for $L$ on the ball $B\left(0,2\|u\|_{L^{\infty}(\Omega)}|| \nabla \theta\left\|_{L^{\infty}(\Omega)}+\right\| \nabla u \|_{L^{\infty}(\Omega \backslash K)}\right)$. This gives

$$
\int_{\Omega} L\left(\nabla u_{k}\right) \leq K_{L} \mid\|\nabla \theta\|_{L^{\infty}}\left\|u * \rho_{k}-u\right\|_{L^{1}}+\int_{\Omega} \theta L\left(\nabla u * \rho_{k}\right)+\int_{\Omega}(1-\theta) L(\nabla u) .
$$

By Jensen Theorem, we have $L\left(\nabla u * \rho_{k}(x)\right) \leq\left(L(\nabla u) * \rho_{k}\right)(x)$. By letting $k \rightarrow+\infty$, we thus get:

$$
\limsup _{k \rightarrow+\infty} \int_{\Omega} L\left(\nabla u_{k}\right) \leq \int_{\Omega} L(\nabla u) .
$$

Since by Fatou Lemma $\liminf _{k \rightarrow+\infty} \int_{\Omega} L\left(\nabla u_{k}\right) \geq \int_{\Omega} L(\nabla u)$, the sequence $\left(L\left(\nabla u_{k}\right)\right)$ converges to $L(\nabla u)$ in $L^{1}(\Omega)$. This completes the proof of Lemma 4.5 .

It is worth noting that as a by-product of the proofs of Lemmas 4.4 and 4.5, we have proved the non occurence of the Lavrentiev phenomenon in the following setting ${ }^{2}$ :

Theorem 4.6. Let $L: \mathbb{R}^{n} \rightarrow \mathbb{R}$ be a convex map of the form $L(\xi)=l(|\xi|)$ for some $l: \mathbb{R}^{+} \rightarrow \mathbb{R}$. Then

$$
\inf _{u \in W_{\phi}^{1,1}(\Omega)} \int_{\Omega} L(\nabla u)=\inf _{u \in \operatorname{Lip}_{\phi}(\Omega)} \int_{\Omega} L(\nabla u) .
$$

\footnotetext{
${ }^{2} \mathrm{~A}$ similar result has been recently obtained by Bonfanti and Cellina with a different method.
} 
Step 4: End of the proof of Theorem 2.5. Let $\alpha \in(0,1)$ and $w \in \operatorname{Lip}_{\phi}(\Omega)$. For the sequence $\left(u_{m}\right)$ given by Lemma 4.5, we write

$$
0 \leq L\left(\alpha \nabla w+(1-\alpha) \nabla u_{m}\right) \leq \alpha L(\nabla w)+(1-\alpha) L\left(\nabla u_{m}\right) .
$$

By the dominated convergence theorem and up to a subsequence (we do not relabel), $\left(L\left(\alpha \nabla w+(1-\alpha) \nabla u_{m}\right)\right)$ converges to $L(\alpha \nabla w+(1-\alpha) \nabla u)$ in $L^{1}(\Omega)$. Hence (4.21) remains true for any $v$ of the form $\alpha w+(1-\alpha) u$, $w \in \operatorname{Lip}_{\phi}(\Omega)$.

Let $w \in \operatorname{Lip}_{\phi}(\Omega)$. For any $k \geq 1$,

$$
k\left(L\left(\nabla u+\frac{1}{k} \nabla(w-u)\right)-L(\nabla u)\right) \leq L(\nabla w)-L(\nabla u) \in L^{1}(\Omega) .
$$

This implies $[\langle\nabla L(\nabla u), \nabla(w-u)\rangle]^{+} \in L^{1}(\Omega)$ and by Fatou Lemma

$$
\begin{aligned}
\int_{\Omega}\langle\nabla L(\nabla u), \nabla(w-u)\rangle-F[u](w-u)= & \int_{\Omega}\left(\limsup _{k \rightarrow+\infty} k\left(L\left(\nabla u+\frac{1}{k} \nabla(w-u)\right)-L(\nabla u)\right)-F[u] \frac{1}{k}(w-u)\right) \\
\geq & \limsup _{k \rightarrow+\infty} k \int_{\Omega}\left(\left(L\left(\nabla u+\frac{1}{k} \nabla(w-u)\right)-L(\nabla u)\right)-F[u] \frac{1}{k}(w-u)\right) \\
= & \limsup _{k \rightarrow+\infty} k\left\{\int_{\Omega} L\left(\nabla u+\frac{1}{k} \nabla(w-u)\right)-F[u]\left(u+\frac{1}{k}(w-u)\right)\right. \\
& \left.-\int_{\Omega} L(\nabla u)-F[u] u\right\} \\
\geq & 0 \quad \text { by (4.21) with } v=\frac{1}{k} w+\left(1-\frac{1}{k}\right) u .
\end{aligned}
$$

We have thus proved

$$
\begin{array}{r}
\langle\nabla L(\nabla u), \nabla(w-u)\rangle \in L^{1}(\Omega) \\
\int_{\Omega}\langle\nabla L(\nabla u), \nabla(w-u)\rangle-F[u](w-u) \geq 0
\end{array}
$$

for any $w \in \operatorname{Lip}_{\phi}(\Omega)$.

By taking $w=(1-\eta) \phi+\eta$ where $\eta \in C_{c}^{\infty}(\Omega)$ is equal to 1 on an arbitrary compact subset of $\Omega$, we get by (4.29) $\langle\nabla L(\nabla u), \nabla u\rangle \in L_{l o c}^{1}(\Omega)$. This implies that $\langle\nabla L(\nabla u), \nabla w\rangle \in L_{l o c}^{1}(\Omega)$ for any $w \in \operatorname{Lip}_{\phi}(\Omega)$. In particular, when $w=(1-\eta) \phi \pm \eta x_{i}\left(1 \leq i \leq n\right.$ and $\eta$ a bump function as above), we obtain $\nabla L(\nabla u) \in L_{l o c}^{1}(\Omega)$.

Now let $\eta \in C_{c}^{\infty}(\Omega)$ and $t>0$. We define $w=\phi+t \eta \in \operatorname{Lip}_{\phi}(\Omega)$. By (4.30), we have

$$
\int_{\Omega}\langle\nabla L(\nabla u), \nabla \eta\rangle-F[u] \eta+\frac{1}{t} \int_{\Omega}\langle\nabla L(\nabla u), \nabla(\phi-u)\rangle-F[u](\phi-u) \geq 0 .
$$

We now let $t \rightarrow+\infty$. This gives

$$
\int_{\Omega}\langle\nabla L(\nabla u), \nabla \eta\rangle-F[u] \eta \geq 0
$$

We then take $w=\phi-t \eta$ to obtain the opposite inequality. Finally, we have

$$
\int_{\Omega}\langle\nabla L(\nabla u), \nabla \eta\rangle-F[u] \eta=0 .
$$

We have thus proved that $u$ is a solution of (E). This completes the proof of Theorem 2.5. 


\section{REFERENCES}

[1] P. Bousquet, The lower bounded slope condition. J. Convex Anal. 1 (2007) 119-136.

[2] P. Bousquet, Local Lipschitz continuity of solutions of non-linear elliptic differential-functional equations. ESAIM Control Optim. Calc. Var. 13 (2007) 707-716.

[3] P. Bousquet, Continuity of solutions of a problem in the calculus of variations. Calc. Var. Partial Differential Equations 41 (2011) 413-433.

[4] F. Clarke, Continuity of solutions to a basic problem in the calculus of variations. Ann. Scvola Norm. Super. Pisa Cl. Sci. (5) 4 (2005) 511-530.

[5] M. Degiovanni and M. Marzocchi, On the Euler-Lagrange equation for functionals of the calculus of variations without upper growth conditions. SIAM J. Control Optim. 48 (2009) 2857-2870.

[6] D. Gilbarg and N.S. Trudinger, Elliptic partial differential equations of second order. Classics in Mathematics, Springer-Verlag, Berlin (2001) Reprint of the 1998 edition.

[7] P. Hartman, On the bounded slope condition. Pac. J. Math. 18 (1966) 495-511.

[8] P. Hartman and G. Stampacchia, On some non-linear elliptic differential-functional equations. Acta Math. 115 (1966) $271-310$.

[9] O.A. Ladyzhenskaya and N.N. Uraltseva, Linear and quasilinear elliptic equations. Academic Press, New York (1968).

[10] C.B. Morrey, Multiple integrals in the calculus of variations. Springer-Verlag, New York (1966). 